\title{
A Fruit Detecting and Grading System Based on Image Processing-Review
}

\author{
Ms. Seema Banot ${ }^{1}$, Dr. P.M. Mahajan ${ }^{2}$ \\ Student, Department of Electronics \& Telecommunication, J.T.M College, Faizpur, India ${ }^{1}$ \\ Professor, Department of Electronics \& Telecommunication, J.T.M College, Faizpur, India ${ }^{2}$
}

\begin{abstract}
This work presents a literature review study for fruit detecting and grading system based on image processing techniques. Different types of algorithms are available to extract feature of fruit characters by capturing the fruit image. With the help of this feature fruit is detected and graded according to quality of fruit this can be done using different types of classifier. The grading system has the advantage of high accuracy, high speed and low cost. It will have a good scene of application in fruit quality detecting and grading areas. This work aimed to study different types of algorithms used for quality grading to developed an algorithm for detecting and sorting of fruit from the acquire image. The feature such as morphological feature is used and colour can be extracted which is further more used to identify class of the fruit using neural network.
\end{abstract}

Keywords: Fuzzy logic Image processing, computer vision, histogram, artificial neural network, fuzzy logic, support vector machine.

\section{INTRODUCTION}

In today's technological era it is necessary to have a good fruit quality for good health of human being, and it is possible by grading the fruits according to size, test, or we can say quality of fruit. But for such grading large man power is required. To overcome this it is necessary to have an automatic fruit grading system for quality fruit production. It is indispensable to have non-destructive automatic quality detection technology in order to improving fruits' quality detection, the system should have grading efficiency and reduce labour requirement. Fruit non-destructive detection is the process of detecting fruits' by each side without damaging the fruit by using some detecting technology to make evaluation according some standard rules. Today it is somewhat difficult to detect fruit shape, size, colour because of poor process, but it is now easy to detect correct fruit by using vision detecting technology. At present, most existing fruit quality detecting and grading system have the disadvantage of low efficiency, low speed of grading, high cost and complexity. So it is significant to develop high speed and low cost fruit size detecting and grading system. In present work we are going to study some technologies used for grading of fruits so as to conclude the best suitable techniques for implementation which fulfils all the necessary requirements.

\section{RELATED WORK}

Machine vision has been mainly used for the quality determination and grading of fruits and vegetables. It has the prospective to automate manual grading processes and minimizes monotonous inspection tasks. Computer vision is also used for defect detection, classification and finding out the ripeness of fruits based on their appearance. This work summarize the review of the various work using different image processing based classification techniques like histogram based method, Fuzzy logic technique, artificial neural network technique(ANN), support vector machine (SVM), Histogram method, RGB color space method, Color mapping technique .

\section{A. Fuzzy Logic Technique}

Ismail Kavdir et al. [1] has applied Fuzzy logic (FL) as a decision making support to grade apples. Quality features such as the color, size and defects of apples were measured through different equipment. The same set of apples was graded by both a human expert and a FL system designed for this purpose. Grading results obtained from FL showed $89 \%$ general agreement with the results from the human expert, providing good flexibility in reflecting the expert's expectations and grading standards into the results. Tajul Rosli et al. [2] proposed and implemented methodologies and algorithms that utilize digital fuzzy image processing, content predicated analysis, and statistical analysis to determine the grade of local mango production in Perlis. The main contribution for this study is on a design and development of an efficient algorithm for detecting and sorting the mango at more than $80 \%$ accuracy in grading compared to human expert sorting. This work proposes a mango grading technique for mangoes quality classification by fuzzy image processing. The method has been implemented using MATLAB language and is suitable for various fuzzy environments. Main advantage of method is the use of fuzzy inference engine without depending on the human expert.

Z. May et al. [3] works on detection of ripeness of oil palm fruit. In this paper, a new system of automatic grading system for oil palm fruit is developed using the RGB color model and artificial fuzzy logic. This automated system uses a computer and a CCD camera to analyze and recognize images. The software code is developed for the image processing part like the segmentation of colors, the calculation of the mean color intensity based on RGB color model and the decision 
making process using fuzzy logic technique to train the data and make the classification for the oil palm fruit. The software code generated has been able to classify the three different classes of oil palm fruit automatically with $86.67 \%$ of overall accuracy.

Suzanawati Abu et al. [4] proposed and implemented Automated Mango Fruit Assessment Using Fuzzy Logic Approach. This work developed a new method of automated mango Size and grade assessment using RGB fiber optic sensor and fuzzy logic approach. They reported that this technique is able to classify the mango into small, medium and large categories .The calculation of maximum, minimum and mean values based on RGB fiber optic sensor and the decision making development using minimum entropy formulation to analyses the data and make the classification for the mango fruit. . The automated mango grading system using fuzzy logic achieved $77.78 \%$ accuracy in overall categories

\section{B. Artificial Neural Network}

Monika Jhuria et al. [5] proposed image processing for smart Farming: detection of decease and fruit grading. artificial neural network is used developed algorithms and they can be successfully detect and classify the tested disease and get better result for color and morphology they reported $90 \%$ result as compared to texture. And also he developed mango grading system depending on weight using mathematical formula weight of mango calculated classify in to 5 different grades.

Nur Badariah et al. [6] proposed an intelligent fruit sorting system using Digital Image Processing and Artificial Neural Networks. For these work apples, bananas, carrots, mangoes and oranges fruits are used. Morphological and color characteristics are used to extract Seventeen Features. Introduction of color features has significantly improved the performance of the system. The classification efficiency has improved to between 79 $90 \%$. Brendon $\mathrm{J}$ et al. [7] proposed the image processing, and neural network classification methods like neural network classifier using wavelets applied to the task of recognizing the pest that causes the damage to apple fruits and leaves in orchards. Author has obtained the good classification rate on a standard neural network without any special alteration to the learning algorithm offers 95 \%recognition rate. Siti Sofiah et al. [8] implemented a simple color identification algorithm using a Neural Network technique and applied to the system to evaluate the ripeness of a banana. The captured image of the banana is resized and its RGB color components are extracted. The color components of the resized images are rescaled using a simple heuristic method. Further, a histogram for the rescaled image is obtained and used as a feature vector to identify the ripeness of the banana. They reported $96 \%$ accuracy using Neural Network classifier with the error back propagation model for ripeness classification is used. Yizhong Wang et al. [9] proposed a non-destructive and evaluating method for fruits based on color recognition. The color images of various fruits were captured and RGB histograms are used as quality features for recognition. A three layer BP neural network is developed wherein its input and output were the RGB histograms and evaluating results, respectively. .
Devrim Unay et al. [10] proposed a technique for apple defect detection and quality classification using MLPneural networks. Here, the analysis of a quality classification system for „Jonagold and „Golden Delicious apples were represented. Later, texture, color and wavelet features are extracted from the apple images. Principal components analysis was applied on the extracted features and some preliminary performance tests were done using single and multi layer perceptrons. The best results were 89.9 and 83.7 per cent for overall and defected pixels of 6 defected images. devrim unay et al.[11] proposed computer vision based system to automatically sort apple. Artificial neural network used for segmentation. 2- Layer, back-propagated network of perceptron neurons (BPNN), which makes binary decision (defected-healthy) for each pixel introduced. Total thirteen features are extracted from each fruit. Linear Discriminant Classifier (LDC), Nearest Neighbor Classifier (k-NN), Fuzzy Nearest Neighbor Classifier (fuzzy k-NN), Adaptive Boosting (AdaBoost), Support Vector Machines (SVM), classifiers was tested for this work. Highest recognition rate is observed with ad boost and support vector machines classifier by $90.3 \%$. Yousef $\mathrm{Al}$ et al. [12] has designed and implemented a prototype computer vision based date grading and sorting system. They have defined a set of external quality features such as flabbiness, size, shape, intensity and defects. The system used RGB images of the date fruits and from these images; it automatically extracted the afore-mentioned external date quality features. Based on the extracted features it classified dates into three quality categories (grades 1,2 and 3) defined by experts using back propagation neural network classifier and tested the accuracy of the system on preselected date samples. The test results showed that the system can sort $80 \%$ dates accurately.

\section{K-Nearest Neighbours Classifier (KNN)}

K-Nearest Neighbors algorithm is also used to identify the input data by comparing it with the trained data. It uses the Euclidean distance measures to measure the distance between points in the input data and trained data [14]

Pragati Ninaws et al. [14] Proposed new fruits recognition techniques with combines four features analysis method. Shape, size and color, texture based method to increase accuracy of recognition. Thirty six fruit images have been collected for fruit recognition system were Twenty fruit images used for training purpose and twelve fruit images are used for testing purpose. For feature extraction they Calculated mean value for RGB component. And shape by threshold segmentation and also Calculate area, perimeter, roundness and entropy values. The recognition result of the accurate up to $95 \%$ using KNN algorithm.

Woo chaw seng et al. [15] proposed in new method for fruit recognition has analyze a, classify and identify fruit image based on the color shape and size feature of the fruit. KNN algorithm is used as classification which is effective classification algorithms the preponed fruit recognized system analyzes and classifies and identifies fruit successfully good accuracy. In this work kNN classifier is used to classify fruit on the bases on mean RGB color value, shape roundness value, area and perimeter value of fruit and they reported $90 \%$ accuracy 
D. Support Vector Machine

Yudong Zhang et al. [16] presented a novel classification method based on a multi-class kernel support vector machine (kSVM) with the required goal of accurate and fast classification of fruits. Number of samples of apple is used for testing the methods. First, fruit images were acquired by a digital camera, and then the background of each image was removed by a split-and-merge algorithm; Second, the color histogram, texture and shape features of each fruit image were extracted to compose a feature space; Third, principal component analysis (PCA) was used to reduce the dimensions of feature space; Finally, three kinds of multi-class SVMs were constructed, i.e., Winner-Takes-All SVM, Max-Wins-Voting SVM, and Directed Acyclic Graph SVM. The experimental results demonstrated that the Max-Wins-Voting SVM with Gaussian Radial Basis kernel achieves the best classification accuracy of $88.2 \%$. For computation time, the Directed Acyclic Graph SVMs performs swiftest.

Suresha M.et al. [17] proposed effective automatic Grading system of apple based on SVM Classifier. The database contains 90 images. The proposed method efficiently classifies apples as red apple and green apple. For these RGB images of apple is converted into HSV image and used threshold based segmentation to segment the apple image form the background. Average red and green color components of the apples are determined for classification of apples. By using linear kernal function for the SVM classifier they reported $100 \%$ accuracy.

Harpreet Kaur et al. [18] proposes machine algorithm to grade the rice kernels using Multi-Class SVM. For this work Indian Basmati rice seed as samples are used for grading and classification. Maximum Variance method was used to extract the rice kernels from background, after that chalk has been extracted from rice. The percentage of head rice, broken rice and brewers rice samples determine by using ten geometric features. Multi-Class SVM classified the rice kernel by examining the Shape, Chalkiness and Percentage of Broken (Head Rice, Broken and Brewers) kernels. The SVM classify accurately more than $86 \%$.

\section{E. RGB Color Space method}

M. Khojastehnazhand et al. [19] presented an efficient algorithm for sorting and grading lemon fruits based on color and size and implemented in visual basic environment. Images of lemon samples were captured by the digital camera are transferred to the PC through the video capture card and then images were digitized, and stored in the PC in RGB (red, green, blue) color space. A program was developed to capture and record the surface images of the lemon. Algorithms were implemented using Visual Basic 6.0 programming language. RGB color space method is used for evaluation of color. Chandra Nandi et al. [20] implemented a computer vision based system for automatic grading and sorting of mangoes based on maturity level from its RGB image frame, collected with the help of CCD camera. Parameters of different classes of mangoes are estimated using Gaussian Mixture Model. Graph contour tracking method based on chain code is adapted for finding the boundary of the mango. This automated technique is good but is further affected by ambient light intensity. Response time of system is on the order of $50 \mathrm{~ms}$. Determination of Size and Ripeness of a Banana has been discussed in [21]. This proposed method emphasizes on the classification of bananas according to its size, shape, texture, color. Three varieties of banana considered for the classification purpose were Mas, Berangan and Cavendish. In terms of size, a banana was classified as extra large (XL), large (L), medium (M) and small (S). Along with the classification, six methodologies of edge detection were discussed, tested and compared in order to get the best method of edge detection. Canny method was proved to be the best one for edge detection among the 6 methodologies. The key idea is the edge detection and the color changes that help in determining the quality of the banana. The area, perimeter, length and thickness of a banana were also determined. Discussion was focused on ripeness percentage. The ripeness percentage was calculated based on the color changes such as when ripe, the fruit turns yellow. The percentage of ripeness can be determined by evaluating the individual pixels of the image.

\section{F. Color Mapping Technique}

Dah-Jye-Lee et al. [22] implemented a Color mapping technique to evaluate the quality and maturity stage of agricultural products like tomatoes and date fruits. Color is used to determine the length of time the tomatoes can be transported and the type of dying process to ripen dates. Color mapping technique converts a specified range of colors of interest in 3-D RGB color space into a smooth \& continuous 1-D color space. It is a simple but effective color grading method. Mr. Unmesh Sagare.et al. [23] implemented a direct color mapping technique and shape to develop a new technique for sorting and grading of fruits .for this work fruit images were captured by using camera and processed by using image processing. Shape features were extracted by calculating area and Major and Minor axis. Direct color mapping concept is converting 3D color spaces to 1-D color indices for automated color grading. It is a simple but effective color grading techniques.

\section{G. HSI technique}

Mohd Z. Abdullah et al. [24] have used the color vision model HSI (Hue, Saturation and Intensity) color space and applied multivariate discriminate analysis to classify oil palms into four quality grades according to PORIM (Palm Oil Research Institute of Malaysia) inspection standards. This work reports misclassification rate of $8 \%$. $P$. Sudhakara Rao et .al [25] have adopted HSI model for sorting and grading of fruits by color and developed a system for on-line sorting of Apples based on color, size and shape. Images are captured by a color CCD camera and frames are separated by a frame grabber card and it produced the image in RGB model. The RGB model, after normalization, is first converted into HSI model using a set of converting equations. The image is analyzed by using advanced image processing techniques to estimate the color of image. By representing median density of Hue as a grading criterion, the image processing system achieved around $98 \%$ accuracy in color inspection of apples. Esehaghbeygi et al. [26] proposed a machine 
vision system for the Evaluation and classification of the Iranian saffron peach. For this experiment Physical features such as size and color were measured to categorize peaches into three quality classes of red-yellow, yellow-red, and yellow the HIS model hue, saturation, and luminance was used for color processing of flawless samples and four boundaries were selected for the peach size image. The size classification accuracy achieved is almost $96 \%$, while the color classification accuracy is around $90 \%$, and that the spot detection algorithm performs well with correct detection levels of $97 \%$ and $85 \%$ for brown and white skin spots, respectively

\section{H. Histogram Method}

Zulham Effendi et al. [27] have developed a Grading System of Jatropha (GSJ) by using color histogram method to distinguish the level of ripeness of the fruits based on the color intensity.

Patrick Jackman et al. [28], proposed a computer vision system for food quality assessment (color, size and shape) using digital camera, PC and other low cost equipment. For extracting surface texture feature of food, he suggested three classical approaches- Pixel Co-occurrence, Run length and Difference Histogram methods. Another option proposed is Fourier Transform which perceives a texture pattern as a convolution of sinusoidal waves. Limitation of this method is it should only be considered where a small number of frequencies can reproduce the surface image. Another alternative to classical approach is Wavelet Transform. It is a perfect method to image texture analysis. He proposed another approach to classical method is Fractional dimension. Author also suggested Artificial intelligence for texture analysis. It is a powerful and robust method.

\section{K- Mean clustering}

Jay Prakash Gupta et al. [29] presented a novel defect segmentation of fruit based on color features with K-mean clustering unsupervised algorithm. K-mean is generally used to determine the natural grouping of pixels present in an image. It is a straightforward and very fast method. Author used defected apples for the testing and evaluated the proposed methods. Proposed method of defect segmentation gives precise results with less computation time. The works proposed earlier using different image processing techniques emphasizes on accuracy of classification of fruits and time to produce the results.

\section{III.CLASSIFICATION TECHNIQUES}

This section describes the most popular and accurate classification techniques that include fuzzy logic, artificial neural network, support vector machine, genetic algorithm technique, histogram based method etc.

\section{A. Fuzzy Logic technique}

Fuzzy logic has been used in a wide range of problem domains. Applications area of fuzzy logic is very wide: process control, management and decision making, operations research, economies and, for this paper the most important, pattern recognition and classification. FL is used to handle uncertainty, ambiguity and vagueness. Once the features are fixed, they are led in input to a classifier which outputs a value associated to the classification of the quality (integer value) or a quality index (real value).

The classification can be divided into two approaches: conventional classification and computational intelligencebased classification. The computational intelligence-based approach includes statistical approach, neural networks and fuzzy systems. It is based on the concept of "partial truth", i.e. truth values between "absolutely true" and "absolutely false". Fuzzy Logic provides a structure to model uncertainty, the human way of reasoning and the perception process. Fuzzy Logic is based on natural language and through a set of rules an inference system is built which is the basis of the fuzzy computation. Fuzzy set theory and fuzzy logic provide powerful tools to represent and process human knowledge in the form of fuzzy IF-THEN rules. A degree of membership became a new way of solving the problems. A fuzzy set is a set whose elements have degrees of membership. An element of a fuzzy set can be full member ( $100 \%$ membership) or a partial member (between 0\% and 100\% membership). That is, the membership value assigned to an element is no longer restricted to just two values, but can be 0,1 or any value in-between. Mathematical function which defines the degree of an element's membership in a fuzzy set is called membership function [30]. Fuzzy logic has many advantages, firstly it is essential and applicable to many systems, moreover it is easy to understand and mostly flexible; finally it is able to model non linear functions of arbitrary complexity. The Fuzzy Inference System (FIS) is one of the main concepts of fuzzy logic and the general scheme is shown in Fig. A FIS is a way of mapping input data to output data by exploiting the fuzzy logic concepts. Fuzzification is used to convert the system inputs, which is represented by crisp numbers into fuzzy set through a Fuzzification function. The fuzzy rule base is characterized in the form of if-then rules and the set of these fuzzy rules provide the rule base for the fuzzy logic system. . Moreover the inference engine simulates the human reasoning process: through a suitable composition procedure, all the fuzzy subsets corresponding to each output variable are combined together in order to obtain a single fuzzy for each output variable.

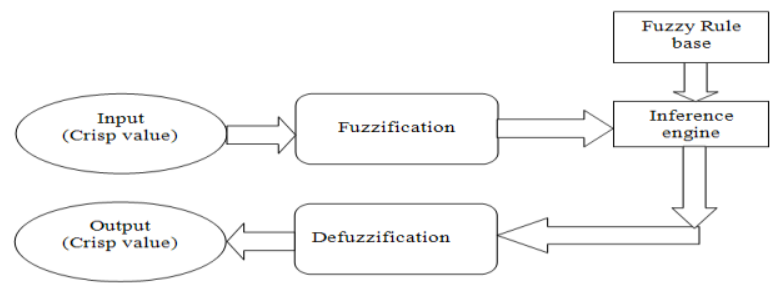

Fig.1. Fuzzy logic concept

Finally the De-Fuzzification operation is used to convert the fuzzy set coming from the inference engine into a crisp value. Fuzzy classification is an application of fuzzy theory. In fuzzy classification an instance can belong to different classes with different membership degrees; conventionally the sum of the membership values of each single instance must be unitary. The main advantage of fuzzy classification based method includes its applicability for very complex processes. The flow of classification using fuzzy logic is described below [31]. 
Input (image channels) and output variables are introduced in Matlab's environment,

- Membership functions are defined using results from supervised classification,

- Matlabs Fuzzy Logic Toolbox was used in definition of fuzzy logic inference rules,

- These rules are tested and verified through the simulation of classification procedure at random sample areas and at the end,

B. Artificial Neural Network

Recently neural networks method has become popular which is used to characterize biological processes. It has best decision-making capability which can be used in image analysis of biological products where the size and shape classification is not achieved by any mathematical function [32]. When it is combined with high-technology handling systems, it gives consistent performance which is the most important benefit of these artificial classifiers in classification of agricultural products [33]. These networks are based on the concept of the biological nervous system, and have proved to be robust in dealing with the ambiguous data and the kind of problems that require the interpolation of large amounts of data. Neural networks have the potential for solving problems in which some inputs and corresponding output values are known, but the relationship between the inputs and outputs is not well understood or is difficult to translate into a mathematical function. These conditions are commonly found in tasks involving grading, sorting and recognizing agricultural products. Figure 2 gives representation of multilayered artificial network.

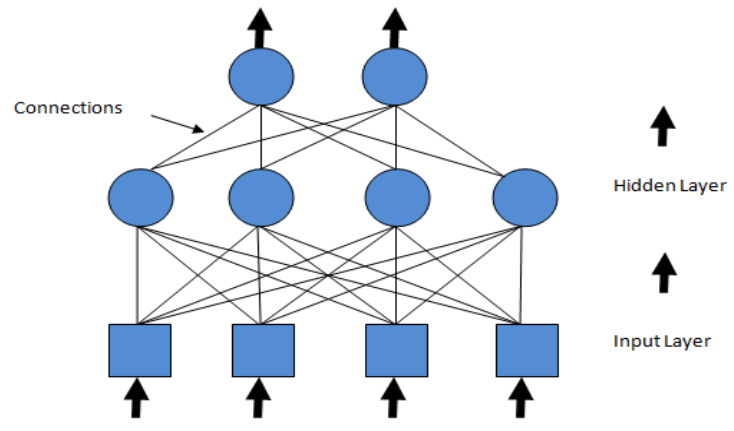

Fig.2. Multi layered artificial network

A neural network is an interconnected group of artificial neurons. In most cases an ANN is an adaptive system that changes its structure based on external or internal information that flows through the network during the learning phase. Modern neural networks are non-linear statistical data modeling tools. They are usually used to solve complex relationships between inputs and outputs or to find patterns in data.

\section{Support Vector Machine}

Support Vector Machines (SVMs) are a supervised learning technique widely used for many different kinds of classification tasks. They were initially conceived to solve classification problems between only two classes, but they can be employed in multi -class problems by using oneagainst -all or one-against-one techniques [32]. In machine learning method, support vector machines (SVMs, also support vector networks) are supervised learning models which consist of learning algorithms that analyze data and identify patterns, used for classification and analysis. Given a set of training examples, each marked as belonging to one of two categories, an SVM training algorithm builds a model that assigns new examples into one category or the other, making it a non-probabilistic binary linear classifier [33]. Figure 3 shows the architecture of SVM.



Fig.3. Principle of Support vector machine

Support Vector Machine (SVM) is a new pattern classifier, set of supervised generalized linear classifiers that have often been found to provide higher classification accuracies than other widely used pattern classification techniques, such as multilayer perception neural networks [9][32]

\section{GRADING TECHNIQUES}

Grading is sorting of vegetables and fruits into different grades according to the size, shape, color, and volume to fetch high price in market. For International market three general grades are considered as:

\section{Extra class \\ 2. Class 1 st \\ 3. Class 2nd}

\section{Extra Class}

The extra class is of superior quality posses the shapes and color of the variety and without internal defect likely to affect the inherent texture and flavor. A 5\% tolerance is allowed for errors. It must be carefully presented taking into accounts the uniformity of the produces in size color, condition arrangement of the produce in the package quality and appearances of the packing or pre-packing material.

2. Class -I

Almost having a same quality is like the Extra Class except that a $10 \%$ tolerance is allowed. Individual fruit is allowed a slight defect in shape, color and miner skin defect which do not affect the general appearance for keeping qualities. In packing the size range may be wider and product need not always be arranged in the package.

3. Class -II

This class product may exhibit some external or internal defects provided they are fit for consumption while fresh. This class is best fitted for local or short distance market. This category will satisfy the needs of customers who are not too demanding and for whom price is more important than quality.

\section{General types of grading}

A) Manual grading

B) Machine vision system for grading 
A) Manual Grading

The method used by the farmers and distributors to sort agricultural products is through traditional quality inspection and handpicking which is time-consuming, laborious and less efficient. Manual grading was carried out by trained operators who considered a number of grading factors and fruit were separated according to their physical quality. Manually grading was costly and grading operation was affected due to shortage of labor in peak seasons.

B) Machine vision system for grading

Grading and sorting machine vision system consist of feeding unite, a belt conveyor to convey the fruit, a color CCD camera located in an image acquisition chamber with lighting system for image capturing, control unite for open and close gates according to signals from computer unite and a computer with an image frame grabber to process the captured image. The acquisition of an image that is both focused and illuminated is one of the most important parts of any machine vision system.

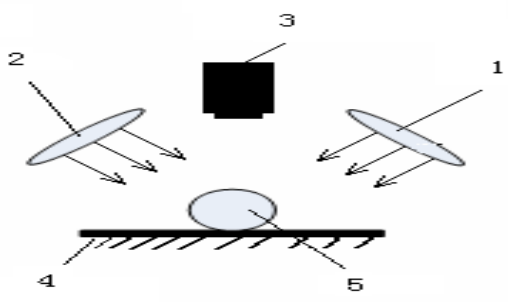

Fig.4. Machine vision system

\section{CONCLUSION}

It is expected from the technology that method can process, analyze and recognize fruits based on color and texture features. In present work we have studied different types of techniques used for fruit detection and grading. Basically this paper gives literature study which includes classifiers using Artificial Neural Network \& Image processing techniques. Also features extraction techniques are studied with different types of grading.

\section{REFERENCES}

[1] Ismail Kavdir, Daniel E. Guyer "Apple Grading Using Fuzzy Logic" Turk J Agric For 27 (2003) 375-382 @ C T.BÜTAK.

[2] Rosli B. Razak', Mahmod B. Othman², Mohd Nazari bin Abu Bakar, Mango Grading By Using Fuzzy Image Analysis, International Conference on Agricultural, Environment and Biological Sciences (ICAEBS'2012) May p.p. 26-27, 2012

[3] Z. May1, M. H. Amaran2, Oil Palm Fruit Grading System using Artificial Intelligence e, International Journal of Video \& Image Processing and Network Security IJVIPNS-IJENS Vol: 11 No: 03, 2011

[4] Suzanawati Abu Hasana, Teoh Yeong Kina, Suraiya Sauddin@Sa'duddina, Nur Badariah Ahmad Mustafa, Kumutha Arumugam, Syed Khaleel Ahmed, Zainul Abidin Md Sharrif “ "Classification of Fruits using Probabilistic Neural Networks Improvement using Color Features"

[5] Monika Jhuria, Ashwani Kumar,Rushikesh Borse ,'image processing for Smart Farming :Detection of disease and Fruit Grading "proceeding of the 2023 IEEE second international conference on image processing (ICIIP-2013)

[6] Nur Badariah Ahmad Mustafa, Kumutha Arumugam, Syed Khaleel Ahmed, Zainul Abidin Md Sharrif "Classification of Fruits using Probabilistic NeuralNetworks - Improvement using Color Features"

[7] Brendon J. Woodford et al, 2010, "Fruit Image Analysis using Wavelets."

[8] Siti Sofiah, "Color Recognition Algorithm using a Neural Network
Model in Determining the Ripeness of a Banana" Proceedings of the International Conference on Man-Machine Systems (ICoMMS) 11 - 13 October 2009, Batu Fer ringhi, Penang, MALAYSIA]

[9] Yizhong Wang et al, 2009, Study on fruit quality measurement and evaluation based on color Identification, Proc. of SPIE Vol. 7513 75130F-1

10] D. Unay, B. Gosselin, 2006, Automatic defect segmentation of Jonagold apples on multi-spectral images: A comparative study, Postharvest Biology and Technology 42 271-279

[11] Devrim Unay, Bernard Gosselin "Artificial Neural Network-Based Segmentation And Apple Grading By Machine Vision"

[12] Yousef Al Ohali, 2011, Computer vision based date fruit grading system: Design and implementation, Journal of King Saud University - Computer and Information Science 23, 29-36.

[13] Kavdır, I., Guyer, D.E.: Comparison of Artificial Neural Networks and Statistical Classifiers in Apple Sorting using Textural Feature,. Biosystems Engg. 89, 331-344 (2004)

[14] Pragati Ninawe1, Mrs. Shikha PandeyA Completion on Fruit Recognition System Using K-Nearest Neighbors Algorithm International Journal of Advanced Research in Computer Engineering \& Technology (IJARCET) Volume 3 Issue 7, July 2014

[15] Woo Chaw Seng,Seyed Hadi Mirisaee" A New Method for Fruits Recognition System"

[16] Yudong Zhang and Lenan Wu, "Classification of Fruits Using Computer Vision and a Multiclass Support Vector Machine", Sensors 2012, Vol: 12, p.p. 12489-12505

[17] Suresha M Shilpa N.A Soumya B “Apples Grading Based On Svm Classifier International Journal of Computer Applications" (0975 8878) On National Conference on Advanced Computing and Communications - Ncacc, April 2012

[18] Harpreet Kaur*, Baljit Singh "Classification and Grading Rice Using Multi-Class SVM International Journal of Scientific and Research Publications, Volume 3, Issue 4, April 20131 ISSN 2250-3153

[19] M. Khojastehnazhand, M. Omid* and A. Tabatabaeefar, Development of a lemon sorting system based on color and size, African Journal of Plant Science Vol: 4, No:4, p.p. 122-127, April 2010

[20] Chandra shekhar Nandi, "An Automated Machine vision based system for fruit sorting and grading", Sixth International conference on sensing technology, 2012

[21] Nur Badariah Ahmad Mustafa, Nurashikin Ahmad Fuad, Syed Khaleel Ahmed, Aidil Azwin, Zainul Abidin, Zaipatimah Ali, Wong Bing Yit, and Zainul Abidin Md Sharrif (2008) 978-1-42442328-6/08 @ IEEE.

[22] Dah-Jye Lee, "Rapid Color Grading for Fruit Quality Evaluation Using Direct Color Mapping", IEEE Transactions on Automation science and Engineering, vol. 8, No. 2, April 2011

[23] Unmesh Shashikant Sagare1, Assoc. Prof. Sunil N. Kore2" Fruits Sorting and Grading Based on Color and Size" International Association of Scientific Innovation and Research (IASIR)(An Association Unifying the Sciences, Engineering, and Applied Research) International Journal of Emerging Technologies in Computational and Applied Sciences (IJETCAS) www.iasir.ne tIJETCAS 12-333; (C) 2013, IJETCAS All Rights Reserved Page 94

[24] Mohd Z. Abdullah et al, 2002, Color Vision System for Ripeness Inspection of Oil Palm Elaeis Guineenszs, Journal of Food Processing Preservation 26 213-235, Malaysia.

[25] P. Sudhakara Rao et al, 2009, Color Analysis of fruits using machine vision system for Automatic Sorting and Grading, J. Instrum. Soc. India 34 (4) 284-291.

[26] A. Esehaghbeygi1*, M. Ardforoushan2, S.A.H. Monajemi3, and A.A. Masoumi1Digital image processing for quality ranking of saffron peachInt. Agrophysics, 2010, 24, 115-120

[27] Zulham Effendi et al, 2009, Development of Jatropha Curcas Color Grading System for Ripeness Evaluation, European Journal of Scientific Research ISSN 1450-216X Vol.30 No.4, pp.662-669

[28] Jay Prakash Gupta, 'Infected fruit part detection using K-means clustering segmentation technique International journal of Artificial Intelligence and interactive multimedia, Vol: 2

[29] Meunkaewjinda A., Kumsawat P., Attakitmongcol K. and Srikaew A. (2008) Proceeding of ECTICON

[30] Nedeljkovic, „Image Classification Based On Fuzzy Logic', The International Archives of the Photogrammetry, Remote Sensing and Spatial Information Sciences, Vol. 34, Part XXX

[31] Chen CH, Ho PP, 2008. "Statistical pattern recognition in remote sensing. J. Pattern Recognition”. 41(9): 4-13.

[32] Kavdir I, Guyer DE, 2008. Evaluation of different pattern recognition techniques for apple sorting. Biosystems Engineering. 99:211-219

[33] Website: http://en.wikipedia.org/wiki/Support_vector_machine 